\title{
RESEARCH IN SENSITIVITY TO ANTIBIOTICS, ANTISEPTICS IN PSEUDOMONAS AERUGINOSA STRAINS ISOLATED FROM PATIENTS WITH INFECTIOUS COMPLICATIONS
}

\author{
O. A. Nazarchuk, D. V. Paliy, N. I. Osadchuk \\ VINNYTSIA NATIONAL PIROGOV MEMORIAL MEDICAL UNIVERSITY, VINNYTSIA, UKRAINE
}

Background. Infections caused by Pseudomonas are one of the topical issues of medicine.

Objective. The aim of the research was to study sensityvity to antibiotics, antiseptics of P. aeruginosa clinical strains that cause infectious complications in patients with burns.

Methods. Microbiological study of biological material, received from 435 patients with burns of the $3^{\text {rd }}$ stages (2011-2015 years). In early terms of burn disease 127 clinical strains of $P$. aeruginosa were isolated from patients. Standard methods were used to identify clinical isolates of $P$. aeruginosa by their morphological, tinctirial, culture and biochemical properties. The research of antimicrobial action of antiseptics, antibiotics against Pseudomonas were carried out by means of standard methods according to the Directive of the Ministry of Health of Ukraine (No. 167 from 05.04.2007 p.) and guidelines of National Committee of Clinical and Laboratory Study (NCCLS, 2002).

Results. It was established that P. aeruginosa caused infectious complications in $23.9 \%$ of patients among other pathogens. Clinical isolates of $P$. aeruginosa were found to be low sensitive to amoxicillin/clavulanate (30.76\%), ceftazidime (25.92\%), cefoperazonum/sulbactam (46.15\%), aztreonam (51.85\%), tobramycin (38.46\%), amicacin (70.34\%), doxiciclini (26.92\%), fluoroquinolones (59.26\%). The analitical progistic criteria of decrease of sensitivity to ceftazidime, cefepim, meropenem and gatifloxacin were found in P. aeruginosa. This pathogen was determined to be sensitive to decasan ${ }^{\circledR}$, antimicrobial composition of decamethoxine ${ }^{\circledR}$, iodine pvidone.

Conclusions. Clinical strains of Pseudomonas aeruginosa, being highly resistant to antibiotics, are also very sensitive to antiseptics decasan ${ }^{\circledR}$, antimicrobial of decamethoxine ${ }^{\circledR}$, povidone iodine.

KEY WORDS: antibiotics; antiseptics; infectious complications; resistance.

\section{Introduction}

Infection is a determinative factor of recovery of patients with different diseases in critical state. It is the main reason of severe complications and mortality among seriously ill patients. Infectious complications, caused by such non-fermenting Gram-negative bacilli as Pseudomonas, are one of the topical matter in contemporary medicine. The incidence of infectious complications, caused by $P$. aeruginosa has no tendency to decrease, despite the use of up-todate methods of diagnostics and treatment of diseases with antimicrobial agents. Management of $P$. aeruginosa related infection is of great significance among burn patients $[1,2]$.

It is established that unsatisfactory results of prevention, treatment of infectious complications of burns are due to distribution of

Corresponding author: Oleksandr Nazarchuk, Department of Microbiology, Vinnytsia National Pirogov Memorial Medical University, 56 Pirogov Street, Vinnytsia, 21018

Phone number: +380432570561

E-mail: nazarchukoa@gmail.com
$P$. aeruginosa polyantibioticresistant strains in surgical clinics. environmental factors Active antimicrobial prevention and management by environmental factors provide selective effect on bacteria, which obtaines new qualities under this action (resistance to environmental factors, high virulence and resistance to antibiotics). Such qualities are determined by genome of microorganisms and can be caused by mutations that prove high adaptation to bacteria. In these cases, infectious complications caused by $P$. aeruginosa are still a current issue $[3,4]$.

Current principles of prevention and treatment of infectious complications in patients with burns include antimicrobials straight after clinical sympthoms are evidenced. So, wide spectrum antimicrobials are empirically used. Nevertheless in conditions of high resistance of microorganisms to antibiotics the absolute therapeutic effect cannot be achieved. Complex approach is one of the most perspective tendency in fight against Pseudomonas infection, 
it include combined use of antibiotics and antiseptics. That is why, the research in the effectiveness of antimicrobials against strains of $P$. aeruginosa that cause infectious complications is essential [5].

The aim of the research was to study sensitivity of $P$. aeruginosa clinical strains, isolated from patients with infectious complications, to antibiotics and antiseptics

\section{Methods}

Microbiological research of wound exudate samples taken from burn surfaces of 435 patients with deep burns of the $3^{\text {rd }}$ stages took place in 2011-2015. All patients enrolled into the research had infectious complications. They were treated in burns centre of N. I. Pirogov Vinnitsya Regional Clinical Hospital. Microbiological research was carried out in bacteriological laboratory of the Department of Microbiology of Vinnytsya National Pirogov Memorial Medical University of the Ministry of Health of Ukraine. The research consisted of isolation of pure culture of pathogens identified by their morphological, tinctirial, culture and biochemical properties. The research samples were separated by sterile plug before antibacterial therapy. 645 strains of opportunistic bacteria were isolated from patients with burn trauma.

During the first 7 days of patients' treatment in the burn centre $P$. aeruginosa caused infectious complications in $23.9 \%$ of cases in comparison with other etiological pathogens. So the sensitivity of $P$. aeruginosa isolates to antibiotics and antiseptics was studied. Biochemical activity of clinical strains of $P$. aeruginosa was defined by means of test-system (NEFERMtest-24 (PLIVA - Lachema a. S., Chech Republic), which consisted of biochemical tests (indol, arginin, urease, lysine, glucose, fructose, inositol, saccharose, phosphatase, beta-galactosidase, beta-glucuronidase, $\mathrm{N}$-acetyl-beta-Dglucosaminidase, mannitol, xylose, cellobiose, galactose, nitrates, nitrites, eskulin, gammaglutamyltransferase, lactose, maltose, tregalose, citrate of Simonce). Cytocromeoxidase activity was additionally studied (OXY-test).

The study of bacteria sensitivity to antibiotics and antiseptics (decasan ${ }^{\circledR}$ (DS), miramistine (MR), chlorhexidine digluconate (CHD), povidon iodine (PI), antimicrobial composition of decamethoxine ${ }^{\circledR}$ (AMC) was conducted by standard methods according to The Directive of the Ministry of Health of Ukraine (No. 167 from 05.04.2007 p.) and guidelines of National Committee of Clinical and Laboratory Study
(NCCLS, 2002). Sensitivity rate of P.aeruginosa clinical strains to antiseptics was evaluated by the value of minimal bactericidal concentration ( $\mathrm{MBcC}$, in $\mathrm{mkg} / \mathrm{ml}$ ) that was obtained by the serial broth dilution method [7].

In the research mathematical and statistical analysis of sensitivity of $P$. aeruginosa clinical strains to antibiotics, antiseptics were conducted. The methods of statistical analysis, used in the research, enabled definition regular relationship between numeric values of variable indications and probability of realization of these values in array observations. To find out the effectiveness of antibiotics current methods of mathematical prediction were used. So, accurate sensitivity of $P$. aeruginosa and its extrapolation on the studied system by building a series of standard normative mathematical models of predicted sensitivity of the pathogen to antibiotics was foreseen. Thus, analytical methods with concretization of absolute and relative optimum meanings were used.

Predictive models of $P$. aeruginosa sensitivity to antibiotics of different groups were investigated as summation of mathematical formulas, which were used to determine these qualities of microorganisms accordingly to their meanings, extrinsic and initial conditions and time. The significance of the results, possible sensitivity rate to antimicrobials was assessed by the coefficient of determination $\left(r^{2}\right)$. The obtained data underwent computer processing by means of original programs "STATISTICA 7"; "Matlab 7.11". For each group of microorganisms strains for each year of study arithmetic mean $(M)$, error arithmetic mean $(m)$, standard deviation $(\sigma)$ were defined. Using the methods of applied mathematics the approximation and interpolation of the data were conducted. Due to this analytic dependence the possible dynamic of sensitivity rate of $P$. aeruginosa strains, isolated from the patients, was estimated [8].

\section{Results}

As the result of the research, 154 clinical strains of $P$. aeruginosa were isolated and identified that looked like typical bacteria of this specimen due to their tinctirial, morphological, and cultural properties. Strains of $P$. aeruginosa, isolated from the patients, had typical biochemical features (Table 1).

All clinical isolates of $P$. aeruginosa (100\%) produced haemolysins, oxidase and decomposed D-mannitol.

As the results of the research, it was proved that $P$. aeruginosa was sensitive to aminopenicillin 
Table 1. Enzymatic properties of isolated strains of $P$. aeruginosa (n 154)

\begin{tabular}{|c|c|}
\hline Test & P. aeruginosa \\
\hline Oxidase & + \\
\hline Catalyse & + \\
\hline D- cellobiose & + \\
\hline Glucose & + \\
\hline y- glutamyltransferase & + \\
\hline D-mannitol & + \\
\hline L1-prolinarylamidase & - \\
\hline Lipase & $+/-$ \\
\hline Urease & + \\
\hline Malonate & $+/-$ \\
\hline Phosphatase & - \\
\hline L- lactate & - \\
\hline D- mannose & - \\
\hline L- histidine & - \\
\hline
\end{tabular}

combined with amoxicillin/clavulanate only in isolated to ceftazidime had not exceeded $12.30-30.76 \%$ of cases (Fig. 1 ).

The study of cephalosporins effectiveness $25.92 \%$ for five years of our study. The efficacy of ceftazidime was decreased by $8.5 \%$ in $2014-$ showed that sensitivity of $P$. aeruginosa clinical 2015 . By means of mathematical analysis the

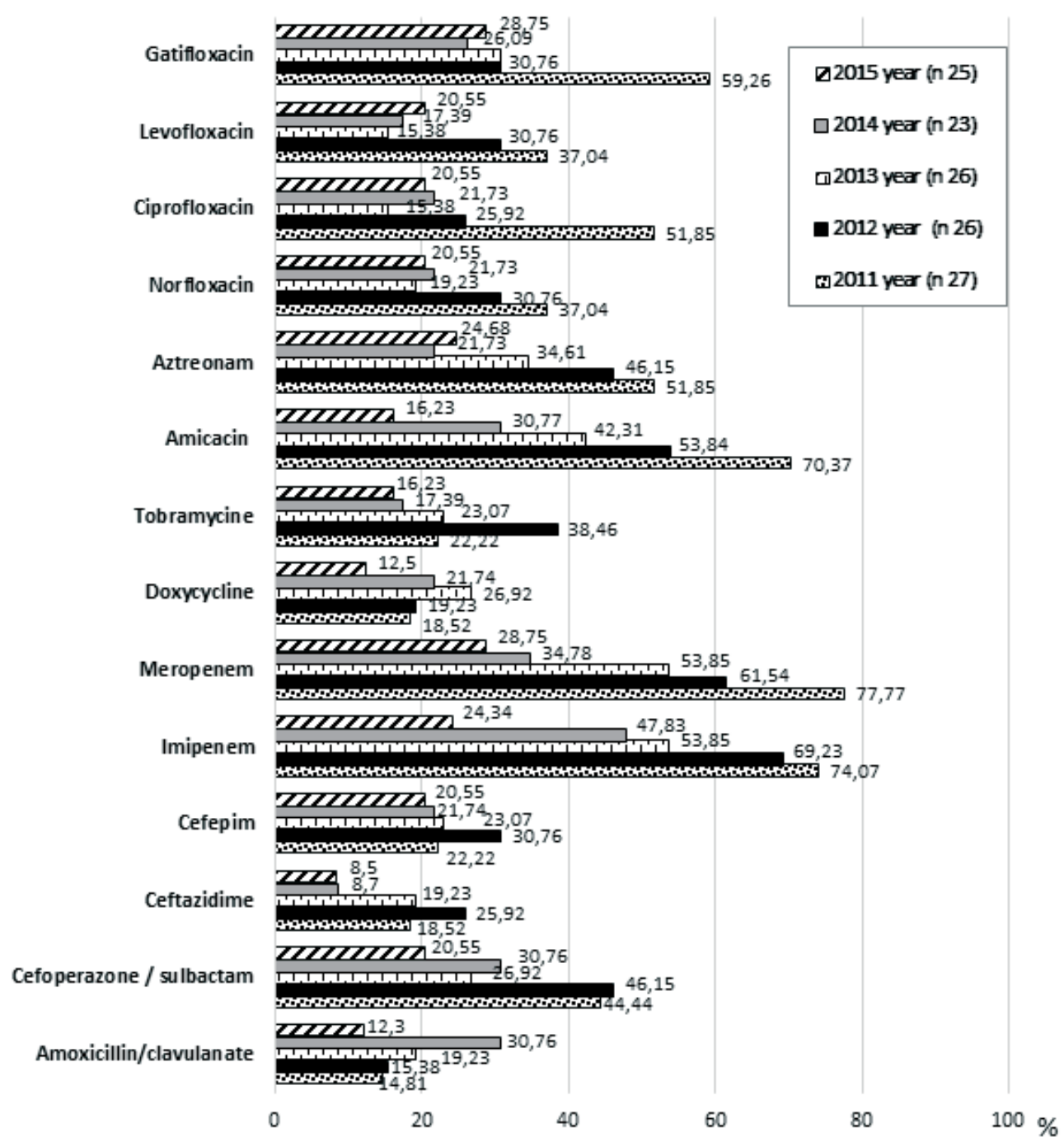

Fig. 1. Sensitivity of $P$. aeruginosa isolates, received from seriously ill patients with burns (\%). 
predicted decrease of $P$. aeruginosa sensitivity to ceftazidime has been proven (Formula 1; Fig. 2).

$$
\text { Ceftazidim }=\frac{1}{a+b x \ln x+c \ln x / x^{2}},
$$

$a=-55358.47 ; b=2.2527981 ; c=1.1111315 e+10$

According to the results of the study of cephalosporins activity, the efficacy of cefepime was not proved (20.55-30.76\%). Prognostic curve of sensitivity of $P$. aeruginosa clinical strains to cefepime could refer to parabolic function, presenting future decrease of the efficacy of this antibiotic. It was found out that $P$. aeruginosa colonized at burn surfaces was resistant to cefalosprins combined with sulbactam. Only $20.55-44.44 \%$ of $P$. aeruginosa clinical strains were sensitive to cefoperazone/sulbactam.

In 2011-2015, the tendency of decrease of $P$. aeruginosa clinical strains sensitivity to meropenem $(28.75 \%)$, imipenem $(24.34 \%)$ was registered. Predictive analysis of $P$. aeruginosa sensitivity to meropenem was revealed by its linear decreasing in 2011-2015 (Formula 2; Fig. 3):

Meropenem $=a+b \times 3+c x / \ln x$, $a=1719659.7 ; b=8.4437694 e-05 ; c=-9101.5317$
Similar decrease of Pseudomonas sensitivity to imipenem was defined in the research.

The estimation of $P$. aeruginosa sensitivity to aminoglycosides proved low efficacy of tobramicin (16.23-38.46\%). The decrease of $P$. aeruginosa sensitivity to amikacin was also evidenced in the research (Formula 3; Fig. 4).

$$
\text { Amikacin } y=a+b x+c x^{2,5}+\frac{d}{x^{1,5}},
$$

$a=-5.8244079 e+9 ; b=2892515.8 ; c=-8.0030078$; $d=1.3157016 e+14 ; x-$ year

Thus, $P$. aeruginosa clinical strains were found to be significantly sensitive to amikacin in 2011 (70.37\%). But in 2015 the number of $P$. aeruginosa sensitive strains was significantly lower (16.23\%). Analytical prediction revealed linear decrease of $P$. aeruginosa sensitivity to amikacin.

It was found out, that isolates of $P$. aeruginosa were of low sensitivity to aztreonam (34.61$51.85 \%$ ). Five-year-study of $P$. aeruginosa sensitivity to aztreonam presented its depression (21.73\%) (Formula 4; Fig. 5).

Aztreonam $y=a+b x 2+c x 4+d x 6$ $a=-6.5573499 e+8 ; b=485.57507 ; c=-0.000119856$; $d=9.861468 \mathrm{e}-12 ; x-$ years

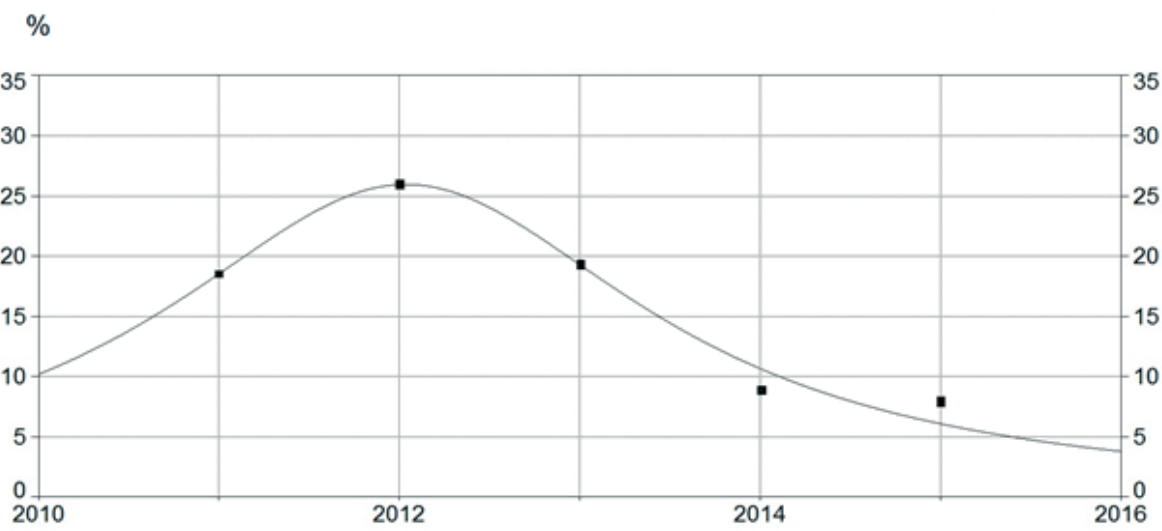

Fig. 2. Predictive sensitivity to ceftazidime of $P$. aeruginosa clinical strains, received from the patients with burn wounds (\%).

$\%$

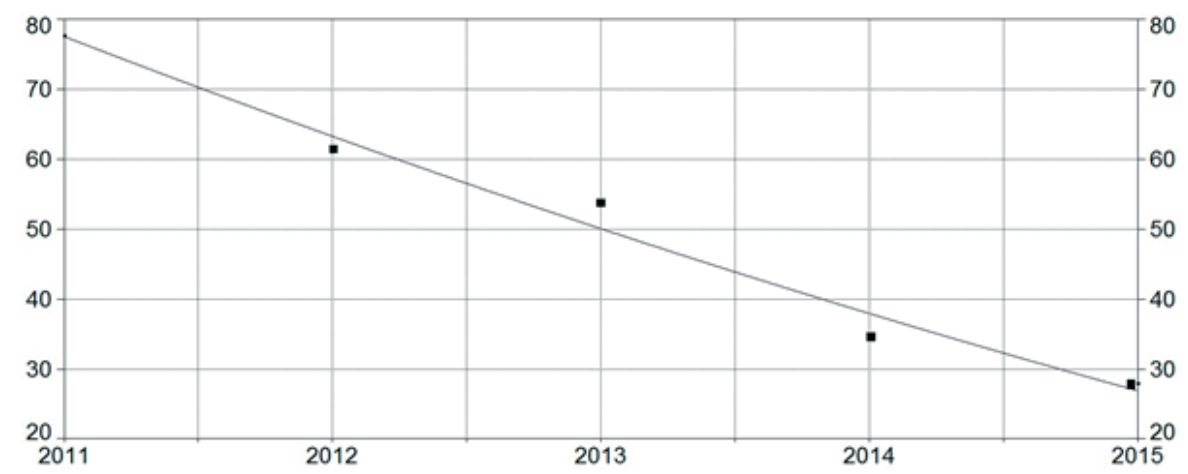

Fig. 3. Predictive sensitivity of $P$. aeruginosa strains to meropenem (\%). 


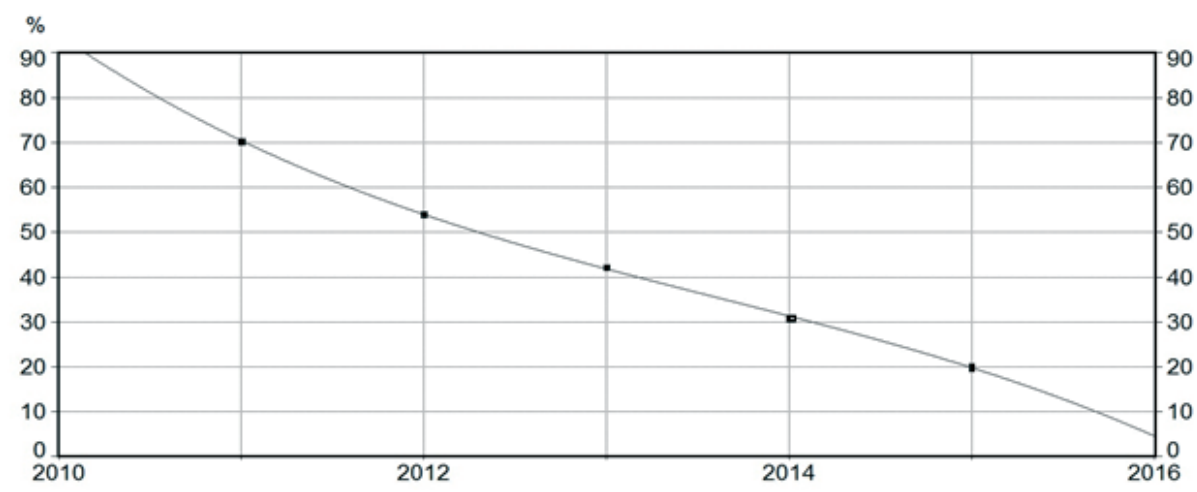

Fig. 4. Predictive sensitivity of $P$. aeruginosa strains to amikacin (\%).

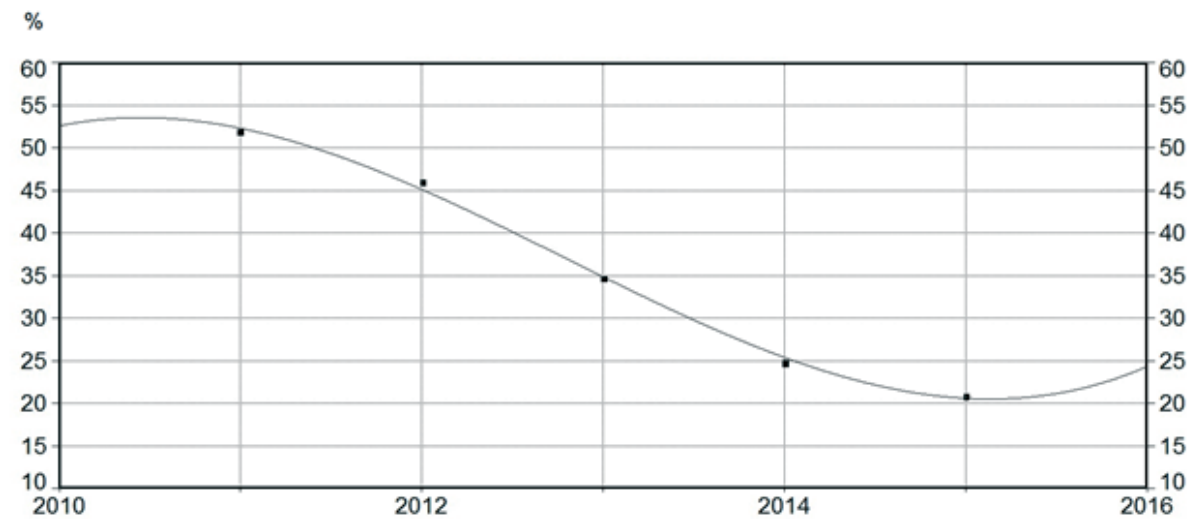

Fig. 5. Predictive sensitivity of $P$. aeruginosa strains to aztreonam.

In $P$. aeruginosa clinical strains of low antibiotic sensitivity, different sensitivity rates to antiseptic remedies were found (Table 2 ).

In the research, clinical strains of $P$. aeruginosa were found to be sensitive to AMC (MBCC $53.15 \pm 1.27 \mathrm{mkg} / \mathrm{ml})$. Inhibitory effect of $D C^{\circledR}$ $(46.65 \pm 0.76 \mathrm{mkg} / \mathrm{ml}), \mathrm{MR}(47.83 \pm 2.04 \mathrm{mkg} / \mathrm{ml})$ did not significantly differ from AMC ( $p>0.05)$. Clinical strains of $P$. aeruginosa were resistant to $\mathrm{ChD}$. Thus, $\mathrm{MBcC}$ of $\mathrm{ChD}$ versus $P$. aeruginosa exceeded $165.85 \pm 10.9 \mathrm{mkg} / \mathrm{ml}$ that proved its low efficacy against these bacteria. $\mathrm{DC}^{\circledR}$ and MR had in 1.8 times higher antimicrobial qualities versus $P$. aeruginosa in comparison with ChD $(p<0.001)$. Antimicrobial activity of AMC was 3 times higher than in ChD ( $p<0.001)$. Antibiotic resistant clinical strains of $P$. aeruginosa were studied to obtain high sensitivity to bactericidal concentrations of povidone iodine that is contained in the official pharmaceutical form of this remedy.

\section{Discussion}

However, in the research the obtained data proved that the amount of isolates of $P$. aeruginosa in general population was high and reached $23.9 \%$ of all microorganisms, isolated from burn wounds in patients, who suffered from infectious complications. Such tendency did not differ from established trend of the increasing importance of P.aeruginosa as a prominent pathogen of infectious complications $[2,5]$.

The results of the research proved low sensitivity of $P$. aeruginosa clinical strains to antibi-

Table 2. Antimicrobial activity of antiseptics against $P$. aeruginos $a$ isolates

\begin{tabular}{|l|c|c|}
\hline \multirow{2}{*}{\multicolumn{1}{|c|}{ Antiseptic }} & $\begin{array}{c}\text { Minimal inhibitory } \\
\text { concentrations }\end{array}$ & $\begin{array}{c}\text { Minimal bactericidal } \\
\text { concentrations }\end{array}$ \\
\cline { 2 - 3 } & \multicolumn{2}{|c|}{$(\mathrm{M} \pm \mathrm{m}), \mathrm{mkg} / \mathrm{ml}$} \\
\hline $\begin{array}{l}\text { Antimicrobial composition of } \\
\text { decamethoxine }^{\circledR} \text { (AMC) }\end{array}$ & $31.0 \pm 0.17$ & $53.15 \pm 1.27$ \\
\hline Decasan $^{\circledR}$ (DC) & $46.65 \pm 0.76^{\dagger}$ & $91.34 \pm 1.69 *$ \\
\hline Miramistin (MR) & $47.83 \pm 2.04^{\dagger}$ & $96.85 \pm 3.25^{*}$ \\
\hline Clorhexidine digluconate (ChD) & $106.3 \pm 4.76^{* *}$ & $165.85 \pm 10.9 * * *$ \\
\hline Povidon iodine & $3858.27 \pm 192.13^{* * *}$ & $6742.13 \pm 397.27 * \star *$ \\
\hline
\end{tabular}

Notes. $\dagger-p>0.05, *-p<0.05 * *-p<0.01, * * *-p<0.001$ in comparison with AMC. 
otics of different groups. The low sensitivity of Pseudomonas to antibiotics, containing betalactam ring in their molecule, was defined. However, in some clinics these antibiotics are widely used as agents of choice for prevention and treatment of infectious complications in patients with burns. Low efficacy of cephalosporins against $P$. aeruginosa according to their sensitivity rate was also determined. Higher sensitivity rate to carbapenems was proved in P. aeruginosa (74.07-77.77\%). The estimation of the antibiotic sensitivity of $P$. aeruginosa to aminoglycosides showed their low effectiveness (16.23-38.46\%). It was proved, that $P$. aeruginosa isolates, received from the patients with deep burns, were of low sensitivity to aztreonam $(34.61-51.85 \%)[3,4]$.

Bactericidal qualities of antiseptics were established that proved high efficacy of decamethoxine, decasan, povidone iodine against clinical strains of $P$. aeruginosa, as pharmaceutical forms contained in adequate doses of these drugs. In the majority of cases bacteri-

\section{References}

1. Church D, Elsayed S, Reid O, et. al. Burn wound infection. Clin Microb Rew. 2006;19:403-434.

2. Nahajchuk VI, Nazarchuk OA, Paliy IG, et al. In addition to characteristics of modern infectious complications in patients with burns. Ukr Med J. 2014;5(103):123-126.

3. Bhandari S, Banjara MR, Lekhak B, et al. Multidrug and pan-drug resistant Pseudomonas aeruginosa: a challenge in post-antibiotic. Nep J of Sc Tech. 2012;13(2):197-202.

4. Chander A, Raza MS. Antimicrobial susceptibility patterns of Pseudomonas aeruginosa clinical isolates at a tertiary care hospital in Kathmandu, Nepal. Ains $\mathrm{J}$ of Pharm and Clin Res. 2013;6(3):235-238. cidal concentrations of miramistin exceeded the dose in the remedy officinal form $[2,6]$.

\section{Conclusions}

Clinical strains of $P$. aeruginosa, which cause infectious complications in seriously ill patients with burn disease, have moderate sensitivity to beta-lactam antibiotics (amoxicillin/clavulanate $-30.76 \%$; ceftazidime $-25.92 \%$; cefoperazone/sulbactam - 46.15\%; meropenem 77.77\%; imipenem - 74.07\%; aztreonam $51.85 \%$ ); aminoglycosides (tobramicin $-38.46 \%$; amikacin - 70.34\%).

Analytical models predict parabolic decrease of sensitivity of $P$. aeruginosa to ceftazidime, cefepime; lineal tendency of decrease of their sensitivity to meropenem, imipenem.

Clinical strains of Pseudomonas aeruginosa, being highly resistant to antibiotics, save their high sensitivity to antiseptics decasan ${ }^{\circledR}$, antimicrobial composition of decamethoxine ${ }^{\circledR}$, povidone iodine. The use of antiseptics is very promising in prevention and treatment of infectious complications caused by $P$. aeruginosa.

5. Frieden T. Antibiotic Resistance Threats in the United States. U.S. Department of Health and Human Services: Centers for Disease Control and Prevention. 2013:114.

6. Paliy GK, Nazarchuk OA, Gonchar OO, et al. The research of physical and chemical, antimicrobial properties of decamethoxin ${ }^{\circledR}$ remedy. Med and clin chem. 2016;18(1):36-44.

7. Nakrasova LS, Svyta VM, Glushkevych TG, et al. The determination of sensitivity of microorganisms to antibacterial drugs: methodological recommendations MR 9.9.5. Kyiv. 2007:74.

8. Yunkerov VI, Grigoryev SG, Rezvantsev MV. Mathematical and statistical processing of medical research data. Saint-Petersburg: VMeedA. 2011:318.

Received: 2016-11-23 